\title{
Drugs: intoxication, safety, adverse events, pharmacogenetics
}

PHARMACOGENETIC AND PSYCHOTROPIC DRUGS

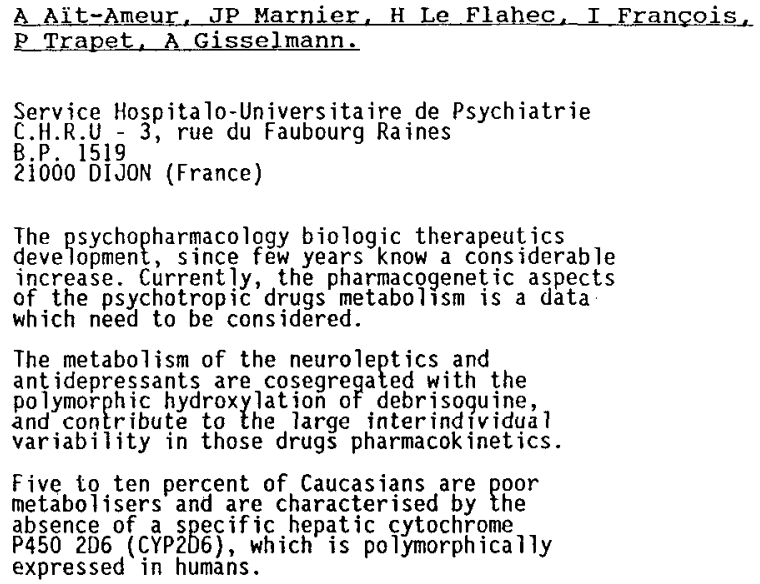

The metabolism of the neuroleptics and ant idepressants are cosegregated with the polymorphic hydroxylation of debrisoguine, and contribute to the large interindividual variability in those drugs pharmacokinetics. Five to ten percent of Caucasians are poor
metabolisers and are characterised by the absence of a specif ic hepat ic cytochrome expressed in humans.

DIFFERENTIAL DIAGNOSIS OF LETHAL CATATONIA AND MALIGNANT NEUROLEPTIC SYNDROME

D. Deltuviene. A.Puras. B. Serksniene

Kaunas Medical Academy, Psychiatry Department, Eiveniu 2, Kaunas 3005, Lithuania

Among the most serious states, treated in the Psychiatry department during the last 5 years, were lethal catatonia $(\mathrm{LK})$ and neuroleptic malignant syndrome (NMS). The clinical picture of those 2 states is very similar, but the treatment is quiet different. For many years DSM-3R and ICD-9 criterions were not available to Lithuanian psychiatrists and our psychiatry was greatly influenced by soviet psychiatry. The adapted version of the ICD-9, used in the USSR and in Lithuania doesn't provide concrete criterions for the diagnosis, in the soviet psychiatry literature they wrote a lot about LK (calling it hypertoxic schizophrenia), did not acknowledge the existence of MNS.

In our work we analyzed 22 cases of the diagnosed LK in 1982-1992 in our psychiatry department, using the criterions of schizophrenia from the DSM$3 R$, evaluating the neurological status and distinguishing lethal catatonia and neuroleptic malignant syndrome according to the onset, initial symptoms, full syndrome, final stage (using the table of the differentiation of LK and MNS from Castillo E., Rubin R.T., Holsboer-Trachsler E.) Those 22 patients with LK were: 9 males, 13 females, age of the patients was 17-57 years, only two of those were admitted to the psychiatry hospital for the first time, others were mentally ill for 3-19 years.

We made a conclusion, that according to the DSM-3R criterions, 6 patients did not provide the criterions of schizophrenia at all, among those 16 patients 11 patients provided a clear NMS and only to 5 patients we left the diagnosis of LK.

There were 12 lethal cases until 1987. From 1987, when we started extracorporal haemosorbtion, there were no more lethal cases, treating both: LK and NMS. 
TREATMENT OF CATATONIA WITH INTRAVENOUS BIPERIDENE

M Franz ${ }^{1}$, B Gallhofer ${ }^{1}$, WT Kanzow ${ }^{2}$

Centre for Psychiatry, Medical School, Justus-Liebig-Universităt Giessen, D-35385 Giessen, Germany

2Psychiatric District Hospital, D-35037 Marburg/Lahn, Germany

Stuporous catatonias are usually treated with high doses of neuroleptics or by electroconvulsive therapy. Treatment with neuroleptics often causes severe side effects which may be similar to catatonic symptoms. Successful treatment of catatonic states with lorazepam and biperidene was reported earlier. The danger of respiratory depression is known to limit the benefit of lorazepam. We studied the effect of biperidene in an open prospective study in 11 patients with catatonic stupor, classified according to DSM-III-R as paranoid type of schizophrenia and schizoaffective disorder. Treatment of catatonic stupor consisted of $5 \mathrm{mg}$ biperidene intravenously, given every 30 minutes up to $15 \mathrm{mg}$. Lack of response required crossing-over up to 3 days with $60 \mathrm{mg}$ haloperidol per day and recrossing-over to biperidene subsequently. The therapeutic effect was measured by means of a rating-scale designed by the authors.

10 patients with catatonic stupor improved within 60 minutes after intravenous biperidene (mean 17,7 minutes, range 4-45 minutes). Doses of biperidene were $5 \mathrm{mg}$ in 8 patients and $10 \mathrm{mg}$ in 2 patients. 1 patient did not improve within 90 minutes following $15 \mathrm{mg}$ of biperidene and was, therefore, treated with 3 days of perfusion of haloperidol $(60 \mathrm{mg} / \mathrm{d})$, however, without success. But catatonic stupor disappeared within 33 minutes due to renewed injection of biperidene

We assume that the evident efficacy of biperidene in stuporous catatonic states is caused by its anticholinergic activity in the basal ganglia. Biperidene binds in competition with acetylcholine to cholinergic muscarinic receptors in the corpus striatum and hippocampus. Spontaneus, reactive or pharmacologically induced states of immobilization and/or catalepsy in various animals can be safely removed by biperidene.

TWO-YEAR POST-MARKETING SURVEILLANCE OF ZOLPIDEM IN SWITZERLAND

Ganzoni $E^{1}$ Santoni JP2

${ }^{1}$ Synthálabo Pharma (CH-1000 Lausanne 21/Switzerland), ${ }^{2}$ Synthélabo (Paris, France).

In Switzerland the Registration Authority Interkantonale Kontrollstelle für Heilmitel (IKS) usually registers New Chemical Entities (NCE) only under condition, called "monitored release". This means that experience with the new drug has to be submitted to IKS annually, including informations regarding the adverse event profile of the NCE.

Zolpidem, a benzodiazepine (BDZ) receptor agonist, with a preferential affinity for the central type $1 \mathrm{BDZ}$ receptor was investigated in a PMS-study covering the first two years after launch in 1990. 1 ' 600 insomniac patients were included in this study, corresponding roughly to $1 \%$ of all units of Zolpidem distributed in Switzerland. The patient characteristics were representative of the general population treated for insomnia (predominantly female in their fifties). 141 (8,8\%) patients experienced adverse events while taking Zolpidem and only $3,6 \%$ required discontinuation of the drug. 185 central nervous system related adverse events and 79 organic symptoms were noted in 141 patients. The events described in $1 \%$ or more were, in decreasing order of frequency, somnolence $(3,3 \%)$, insomnia $(1,8 \%)$, vertigo/nausea $(1,8 \%)$, dreaming $(1,3 \%)$, confusion ( $1 \%)$; neurologic symptoms (headache) $(1,4 \%)$ and gastrointestinal symptoms (abdominal pain, vomiting) $(1,0 \%)$. The adverse event profile reflects the official labeling of zolpidem and its pharmacological properties and is consistent with the cumulative international experience on the drug. No previously unrecognized safety issues were identified. Thus, the favourable safety profile of zolpidem, when administered in harmony with the prescribing information, is confirmed.
AKATHISIA: POINT - PREVALENCE OF THE SYNDROME IN AN INPATIENT POPULATION IN AIHENS

B J Havaki-Kontaxaki, V_P Kontaxakis, G N Christodoulou and C N Stefanis

Center for Mental Health, Athens

Department of Psychiatry, University of Athens,

74 Vas. Sophias Ave, 11528 Athens, Greece

Akathisia is a syndrome of motor restlessness that occurs as an adverse effect of treatment with antipsychotic drugs. The aim of this prospective study is the estimation of the pointprevalence of akathisia cases in the inpatient population of two wards (one male and one female) in a long-stay psychiatric hospital. The survey took place at Psychiatric Hospital of Attica (PHA), Athens. A total of 164 patients from two wands (one male, one female) of the PHA were studied (105 men and 57 women). The diagnostic categorization of the patients was carried out by two independent psychiatrists on the basis of DSM-IIIR criteria. Schizophrenic disorder was diagnosed in most of the patients (718). Other diagnoses of the patients were the following: organic delusional disorder (128), bipolar mood disorder (8\%), schizoaffective disorder (48). For every patient a standardized questionnaire containing sociodemographic and clinical data was completed. All patients were examined by a team of psychiatrists - assessors ( $N=6$ ) specially trained in the recording of the Akathisia symptcmatology. Akathisia was assessed using the Barnes Akathisia Rating Scale (1989). Akathisia was diagnosed if the score on the global item of the BARS was 2 or more. During the time period of the survey 27 patients (16.58) had developed Akathisia. Among them 20 patients were male(19\%) and 7 patients were female $(12.38)$ The relationships between akathisia and many clinical and demographic variables are examined.

MOCLOBEMIDE - CLINICAL SAFETY - - FROM CLINICAL TRIALS TO THE WORLD MARKET

Susan Hilton, Birgit Jaber, Rose Ruch, Eliane Goertz Pesenti. Drug Safety Department. F. Hoffmann-La Roche Ltd. CH-4002 Basel, Switzerland

Moclobemide is a reversible inhibitor of monoamine oxidase $\mathrm{A}$. That it is a monoamine oxidase inhibitor (MAOI) means that stereotypes exist in expectations with this new product, based on experience with 'old' MAOI's. This paper presents the product safety profile after 3 years on the world markets. Roche Drug Safety collected data on adverse experiences regardless of causality $(\mathrm{AE})$ from all sources worldwide, through compound development and after launch. In short-term ( $<12$ weeks) clinical trials, AEs significantly more frequent with moclobemide than with placebo were dizziness, nausea and sleep disturbances only. Compared to tricyclic antidepressants (TCA, usually imipramine), AEs known for TCA (dry mouth, sweating, blurred vision, dizziness, tremor) and somnolence occursed significantly more often with TCA than with moclobemide. Insomnia and headache were more common with moclobemide.

To 30 June 1993, 1387 patients from an estimated exposed population of 750,000 reported 2390 AEs of which 1335 (55.7\%) were considered attributable to moclobemide. Psychiatric disorders (28.4\%, not surprising when indications for moclobemide use are considered), CNS AEs (16\%) and gastrointestinal AEs (13.6\%) were most frequent. Other AEs related to body-as-a-whole $(9.2 \%)$, skin $(5 \%)$, general cardiovascular $(4.7 \%)$ and all other body systems combined ( $23.1 \%$ ). AEs typical for the 'old' MAOI were rare; only $1.1 \%$ hepatobiliary AEs, suggesting little or no hepatotoxic potential, blood pressure changes reflecting background prevalence of disease and no proven tyramine interaction. Roche Drug Safety monitoring shows moclobemide to have a safety profile quite different from TCA and 'old' MAOI and to be a safer antidepressant. 
MOCLOBEMIDE AND CARDIAC SAFETY

Susan Hilton, Birgit Jaber, Rose Ruch, Eliane Goertz Pesenti.

Drug Safety Department, F. Hoffmann-La Roche Ltd., CH-4002 Basel, Switzerland

Moclobemide is a reversible and selective inhibitor of monoamine oxidase subtype A with a wide spectrum of antidepressant activity. So as to fully evaluate product safety, Roche Drug Safety has collected data on adverse experiences (AEs) regardless of causality from all sources worldwide through compound development and after launch in 1989. Review was made of all general cardiovascular, myocardial, heart rate and thythm disorders reported from all sources worldwide to 30 June 1993 in order to assess the effect (if any) of moclobemide on cardiac function. This review revealed that 176 patients from an estimated exposed population of 750,000 reported 203 AEs of which 79 AEs $(38.9 \%$ ) were considered attributable to moclobemide. In all $16 / 176$ patients $(5.3 \%)$ died. All fatalities were the consequences of endstage cardiovascular disease. Analysis of the results showed that the disorders reported were attributable to pre-existing cardiovascular disease, cardiovasculotoxic concomitant therapy, smoking, alcohol or other drug abuse, intentional drug overdose or anaphylactoid reaction. The available data do not, therefore, indicate an adverse effect of moclobemide on cardiac function.

MOCLOBEMIDE AND LIVER DISEASE

Susan Hilton, Birgit Jaber, Rose Ruch, Eliane Goertz Pesenti.

Drug Safety Department, F. Hoffmann-La Roche Ltd., CH-4002 Basel, Switzerland

Moclobemide is a reversible and selective inhibitor of monoamine oxidase subtype A with a wide spectrum of antidepressant activity, first launched in 1989. Many of the established antidepressant drugs have been found to be hepatotoxic when used in animal models. Traditional monoamine oxidase inhibitors (MAOI's) may have a greater potential for hepatotoxicity than seen with the tricyclic antidepressants. Phenelzine has been linked to the development of hepatic necrosis, and shows cross-toxicity with other chemically-related MAOI's (Schăppli, 1985). Moclobemide appears to be completely devoid of potential hepatotoxicity in animal models (Schappli, 1985; Burkard et al., 1992). The metabolic patterns in urine and bile of rat and man (Schoerlin and Da Prada, 1990) are largely similar, and it therefore seems rather unlikely that moclobemide may produce a toxic metabolite in man. Roche Drug Safety collected clinical safety data on adverse experiences regardless of causality (AEs) from all sources worldwide, through compound development and after launch in 1989. To 30 June 1993, 1387 patients from an estimated exposed population of 750,000 reported 2390 AEs of which 1335 AEs $(55.7 \%)$ were considered attributable to moclobemide. Only 27 hepatobiliary AEs $(1.1 \%$ of total AEs) were reported, most of which were considered minor changes in hepatic enzymes or infectious hepatitis unrelated to moclobemide. Analysis of these clinical results suggests that moclobemide has little or no hepatotoxic potential, in agreement with animal studies.
METABOLISM OF TRICYCLIC ANTIDEPRESSANTS IN PATIENTS

H.-J. Kuss, D.Y. Zhao, S. Sirch, M. Ackenheil Department of Neurochemistry, Psychiatric Clinic of the University of Munich

Nussbaumstr. 7, D-80336 Munich, Germany

All clinically used tricyclic antidepressants are metabolized by demethylation, hydroxylation and subsequent glucuronidation. The demethylation ratio can be important for the therapeutic efficacy as pharmacological properties of the metabolite may be different. Due to structural differences the mean ratio desmethyl compound : mother substance is different for each antidepressant drug. The mean ratio for chlorimipramine is $2.07 \pm 1.13$ $(n=247)$, but it is $0.68 \pm 0.42(n=432)$ for maprotiline. Patients in their demethylation ratio show great interindividual, but small intraindividual variations in each drug. The ratio is influenced by neuroleptic or other comedication, the effects being different in the different anti-depressants. In a group of 131 patients receiving maprotiline and chlorimipramine simultaneously, plasma concentrations of both drugs as well as of their desmethyl metabolites were measured by HPLC. One would assume, that patients with a high demethylation (concentration of the mother substance divided by the dose) for one tricyclic also have a high demethylation ratio for another tricyclic drug. This is not the case, as could be shown in this group of patients. We found a similar result in a group of 28 patients under treatment with amitriptyline and chlorimipramine. Knowing the plasma concentrations and the demethylated metabolite for one tricyclic drug does not enable to predict plasma concentrations for another antidepressant drug. The metabolism of tricyclic drugs seems to be more complex. With regard to the above results it seems very critical to phenotype patients by tests using chemically different drugs. Knowledge of the isoenzyme specific metabolism may explain some major regularities, but does not help the individual patients.

CONTRIBUTION OF DRUG INTERACTIONS TO THE PHARMACOKINETIC VARIABILITY OF NORTRIPTYLINE OBSERVED UNDER THERAPEUTIC DRUG MONITORING K Linnet

Institute for Basic Research in Psychiatry, Clinical Biochemical Laboratory, Psychiatric Hospital in Aarhus, Skovagervej 2, DK-8240 Risskov, Denmark.

In this study we evaluated the impact of drug interactions on the variability of the steady-state concentration/dose ratio (C/D) for nortriptyline as observed under therapeutic drug monitoring. The concentration/dose ratio was recorded for a total of 611 consecutive patients. Serum concentrations of nortriptyline were determined by HPLC. The median value of $C / D$ was 4.10 , and the total range was from 0.4 to 21.0 (nmol/L/mg daily dose). $167(27 \%)$ of the patients received drugs that are able to accelerate or inhibit the metabolism of nortriptyline, e.g. antiepileptics, neuroleptics, selective serotonin reuptake inhibitors and cimetidine. Omitting these patients, the median C/D of the remalning 444 patients was 3.95 , and the total range extended from 0.4 to 21.0 (nmol/L/mg daily dose). The variability of C/D was expressed as a nonparametric index of dispersion equal to 1 $=(90$-percentile -10 -percentile)/median. $I$ was 1.43 for the $\mathrm{C} / \mathrm{D}$ of the total group of 611 patients and 1,47 for the 444 patients who did not receive potentially interacting medicine.

In conclusion, other factors than drug interaction are responsible for the large variability of $C / D$ of nortriptyline observed under routine therapeutic drug monitoring.

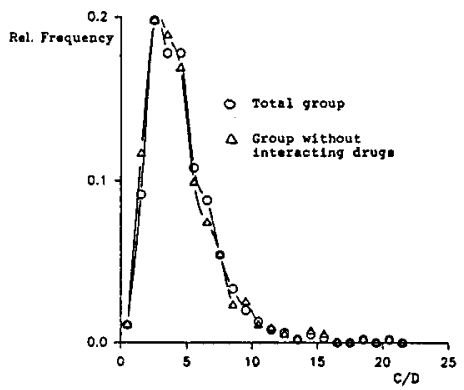


THE SEARCH OF EVALUATION CRITERIA OF MINIMAL DOSES RADIATION EFFECTS ON PSYCHIATRIC PATIENTS

SA Malyarov, IV Krook, VA Procic

Ukrainian State Medical University, Shevchenko blvd. 13, 252004, Kiev, Ukraine.

The goal of the investigation is the objective clinic estimation of minimal radiation doses inducing psychic disorders changes. The comparation of histories in 2 groups of 140 psychotic patients each was fulfilled. All patients were Kiev residents. The lst group consists of persons hospitalized within the year after Chernoby 1 disaster in 1986, the 2nd group consists of persons hospitalized' in 1985. Clinic and psychometric methods were used. The original psychodiagnostic system for indication of asthenic states was created. The frequencies of asthenic and hypochondric complaints in both groups were equal. It seems impossible to make reliable clinic differentiation of social, personality and biological causes of those complaints. It was found out however, that hypersensitivity to small doses of antipsychotics and non-reflected in complaints exhaustion are more frequent in 1 st group of patients. So these signs have a diagnostic value for clinic indications of radiationinduced psychic disorders changes.
ORAL BENZODIAZEPINES - SAFETY: 20 YEARS OF EXPERIENCE Rose Ruch, Birgit Jaber, Susan Hilton, Eliane Goertz Pesenti.

Drug Safety Department, F. Hoffmann-La Roche Ltd., CH-4002 Basel, Switzerland

Since the early 1970s Roche Drug Safety has collected worldwide all spontaneously reported cases of adverse experiences (AEs) associated with oral benzodiazepines (BZD) regardless of causal relationship. In patients receiving diazepam, bromazepam, midazolam, flunitrazepam, flurazepam and nitrazepam in recommended doses, a total of 4588 cases with 6713 AEs ( 3400 or $50.7 \%$ rated attributable), have been entered onto the Drug Safety database up to 31 October 1993 . A total of 976 cases with 1385 AEs (20.7\%) were rated 'serious' as defined by the Council for International Organizations of Medical Sciences (CIOMS). In all, 151 patients (3.3\%) have died. AEs were classified according to System Organ Class (SOC) of the World Health Organization (WHO). The analysis of the figures shows that $54.8 \%$ of all AEs are psychiatric or CNS disorders. This is not surprising considering indications for the oral BZD. Less commonly reported were skin disorders $542(8.1 \%)$ of which 199 AEs were associated with bromazepam; body-as-a-whole disorders $\$ 14$ (7.7\%) gastrointestinal disorders $407(6.1 \%)$; respiratory disorders $214(3.2 \%)$; liver and biliary disorders $165(2.5 \%)$; vision disorders $143(2.1 \%)$ special senses disorders $96(1.4 \%)$ of which 77 taste alterations were associated with flurazepam. All cardiac and cardiovascular disorders combined amounted to $3.8 \%$ and all blood disorders to $3.2 \%$. All other SOC amounted to $7.1 \%$. Cardiac, respiratory, gastrointestinal and skin disorders are rare; liver disorders, blood dyscrasias and all other AEs occur in isolated cases. This is summed up in the conclusion drawn in Goodman and Gilman's, The Pharmacological Basis of Therapeutics, 8th Ed., 1990, p.356: "In spite of the adverse effects reviewed above, the BZD are relatively safe drugs".
ORAL BENZODIAZEPINES - SAFETY IN OVERDOSE; 20 YEARS OF EXPERLENCE

Rose Ruch, Birgit Jaber, Susan Hilton, Eliane Goertz Pesenti.

Drug Safety Department, F. Hoffmann-La Roche Ltd., CH-4002 Basel, Switzerland

Since the early 1970s Roche Drug Safety has collected worldwide all spontaneously reported cases of overdoses associated with oral benzodiazepines (BZD). A total of 1771 case reports of patients taking overdoses of diazepam, bromazepam, midazolam, flunitrazepam, flurazepam or nitrazepam alone or in combination with other CNS. depressants, analgesics and/or alcohol have been entered onto the Drug Safety data base up to 30 September 1993. A total of 340 patients $(19.2 \%)$ have died. All main effects observed in these patients were those expected from the pharmacology of oral BZD or the concomitantly taken substances. In all, 981 patients were reported to have taken one of the oral BZD alone, of which $98(10 \%)$ have died. A number of these patients who died suffered from serious underlying conditions, e.g. respiratory or cardiac disorders, diabetes mellitus, alcohol and drug abuse. A total of 790 patients are known to have taken other CNS-depressants, analgesics or alcohol in any combination concomitantly with the oral BZD. Of these patients 242 $(30.6 \%)$ died. These figures are broken down for the individual oral BZD as follows:

\begin{tabular}{lrrrrr}
\hline Oral BZD & All cases & Died & BZD alone & Died & $\%$ All cases \\
Diazepam & 273 & 54 & 105 & 9 & 3.3 \\
Bromazepam & 301 & 37 & 182 & 11 & 3.7 \\
Midazolam & 55 & 13 & 33 & 4 & 7.3 \\
Flunitrazepam & 583 & 77 & 386 & 26 & 4.5 \\
Flurazepam & 317 & 84 & 153 & 20 & 6.3 \\
Nitrazepam & 242 & 75 & 122 & 28 & 11.6 \\
TOTAL & 1771 & 340 & 981 & 98 & 5.5 \\
\hline
\end{tabular}

This indicates that overdoses of oral BZD are rarely fatal unless other CNS-depressants, analgesics and/or alcohol ase taken concomitantly. 